\title{
Performance evaluation and mathematical analysis of direct sequence and frequency hopping spread spectrum systems under wideband interference
}

\author{
Fawzan Ghalib Abdul Karim Bawahab a,1, Edi Kurniawan ${ }^{\text {b,2, }}{ }^{\text {, }}$, Elvan Yuniarti ${ }^{a, 3}$, Bambang \\ Widiyatmoko ${ }^{\mathrm{b}, 4}$, Dwi Bayuwati ${ }^{\mathrm{b}, 5}$ \\ ${ }^{a}$ Department of Physics, UIN Syarif Hidayatullah, Tangerang Selatan, Indonesia \\ ${ }^{b}$ Research Center for Physics, Indonesian Institute of Sciences (LIPI), Tangerang Selatan, Indonesia \\ ${ }^{1}$ fawzanghalib@gmail.com; ${ }^{2}$ edi.kurniawan@lipi.go.id; ${ }^{3}$ elvan.yuniarti@gmail.com; ${ }^{4}$ bambang.widiyatmoko@lipi.go.id; \\ ${ }^{5}$ dwi.bayuwati@lipi.go.id \\ * corresponding author
}

\section{ARTICLE INFO}

\section{Article history}

Received August 10, 2018

Revised September 17, 2018

Accepted October 4, 2018

Available online November 11, 2018

Keywords

DSSS

FHSS

Spread spectrum

Interference

Bit error ratio (BER)

\section{ABSTRACT}

This paper presents performance evaluation and comparison analysis of Direct Sequence Spread Spectrum (DSSS) and Frequency Hopping Spread Spectrum (FHSS) systems. The evaluation and analysis are done based on the systems performance against wideband interferences. The interferences are signals with similar spectrum characteristic to the transmitted signals of DSSS and FHSS systems. Bit Error Ratio (BER) is used as evaluation parameter to assess the performance of both systems. Simulation and mathematical analysis are performed to test and verify the performance of both systems. Mathem atical analysis also verifies that increasing Spreading Frequency on certain conditions will reduce the BER. This research also points out that FHSS system has a better performance compared to DSSS system indicated by smaller BER.

This is an open access article under the CC-BY-SA license.

\section{Introduction}

Spread Spectrum Multiple Access (SSMA) is one of the Multiple Access techniques in communication systems, that works by expanding the transmitted signal bandwidth to be larger than the bandwidth of the data signal [1]. Previous works discussing theory [2], techniques [3], and the developments of Spread Spectrum system [4] have been published since three decades ago. Spread Spectrum was originally applied to military communication system [5]. Currently, Spread Spectrum techniques have been widely used in many communication systems [6] such as GLOBALSTAR satellite communications system [5], Low Earth Orbiting (LEO) satellite communications network systems [7], GPS for satellite navigation system [8], Wireless Local Area Networks (WLAN) IEEE 802.11 system [8], optical codes for Fiber-optic Local Area Networks (LAN) [9], digital cellular radio system IS-95 for mobile voice communication [8], Wideband-CDMA for third-generation mobile communication system [8], wireless communication and control systems in Unmanned Aerial Vehicles (UAV) [10], underwater acoustic communication system [11]. This spread spectrum system is now developed for multiple access techniques on fifth generation (5G) cellular telecommunication system [12]. Spread Spectrum systems have been used in many other applications such as digital watermarking for copyright protection of audio, image and video, and multimedia data [13]-[15], digital watermarking for telemedicine [16], DC / DC converter circuit [17], LLC Resonant [18], and 3rd Generation Attachment Series (SATA-III) for reducing electromagnetic interference (EMI) problems [19]. 
Spread Spectrum systems have been used in various fields due to their characteristics that can solve interference problems. Characteristics of the Spread Spectrum systems offering advantages for communication systems have been described in [20]. Simulation study by using Matlab software as a digital signal processing tool has been done in [21]. The advantages of the Spread Spectrum systems compared to the FDMA and TDMA systems have been ddiscussed in [22]. In general, there are two methods of Spread Spectrum, namely: Direct Sequence Spread Spectrum (DSSS) and Frequency Hopping Spread Spectrum (FHSS). Several works addressing a thorough study of those two systems have been done in [23-30]. In [23] and [24], design and simulation of both systems have been done on Matlab platform. In [25], a comparison study was done by changing the used signal bandwidth. Performance analysis by varying the pseudo noise (PN) sequence and modulation technique has been conducted in [26]. In [27], comparative analysis based on the resulting modulated signal form has been presented. In [28], both systems are analyzed for their ability to avoid interference based on the maximum number of users for the same receiver. McCune [29] and Shayesteh [30] studied the performance of Spread Spectrum systems against interference with narrowband frequency.

This research aims to simulate and analyse DSSS and FHSS performances when both systems are subject to wideband interference. The comparison analysis will be done based on the robustness of both systems against this type of interference. The used interference signal has similar spectrum behaviour to the transmitted signals of DSSS and FHSS systems. In other words, the generated interference signal employs similar frequency channel to DSSS and FHSS systems. BER parameter is used to determine the ability of each system to recover the data signal. This performance evaluation and mathematical analysis are important in the development of new multiple-access techniques, which are more resistant to wideband interference.

This paper is structured as follows; Section 1 introduces the principle, the applications, and the previous works on Spread Spectrum system. Section 2 describes an overview of Spread Spectrum System covering the signal processing techniques on DSSS and FHSS systems, and presents the methodology covering the design procedure for simulation. Section 3 describes the simulation results and mathematical analysis, then followed by remarks. Section 4 concludes the paper.

\section{Method}

\subsection{Overview}

Spread Spectrum is one of the multiple-access techniques that operates by expanding the transmitted signal bandwidth to be larger than the data signal [1]. Spread Spectrum systems are generally divided into two techniques: DSSS and FHSS. DSSS transmits signals at a single frequency but on very wide bands, while FHSS transmits signals with narrow bands, but quickly jumps from one frequency to the next. Fig. 1 illustrates the difference between the spectrum of DSSS and FHSS.

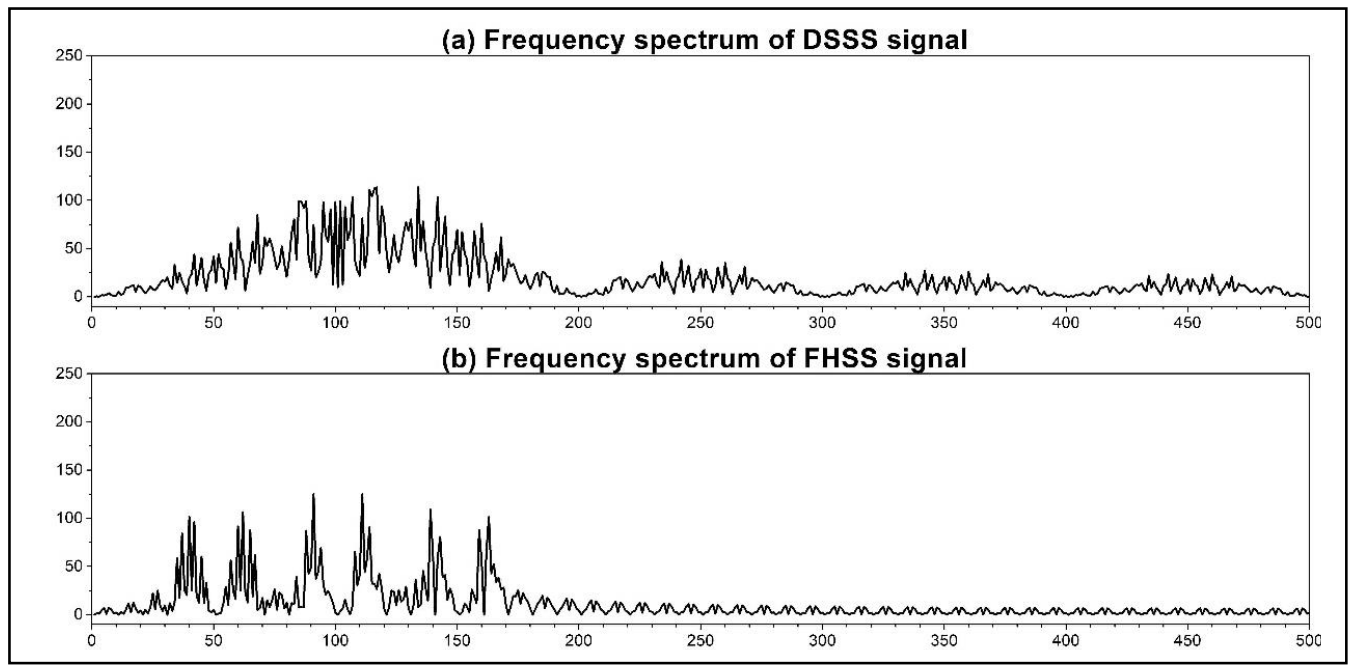

Fig. 1. Frequency spectrum of (a) DSSS \& (b) FHSS 


\subsubsection{Direct Sequence Spread Spectrum}

DSSS is most widely used type of Spread Spectrum, due to it is easier to implement and also has a high data rate. DSSS is a method to transmit data where the sending and receiving systems are on a wide frequency band. This wide channel allows the device to transmit more information at a higher data rate. Fig. 2 shows the signal processing steps on both transmitter and receiver of DSSS system. DSSS works by modulating the data signal with the carrier signal, then spreading it with a spreading signal (pseudorandom signal) whose frequency is greater than the frequency of the data signal (bitrate). In the receiver, the despreading signal is generated from multiplication by the carrier signal and the same spreading signal on the transmitter, reversing the encoding process on the transmitter [3]. The last process on the receiver is filtering to eliminate other signals, while keeping data signal.

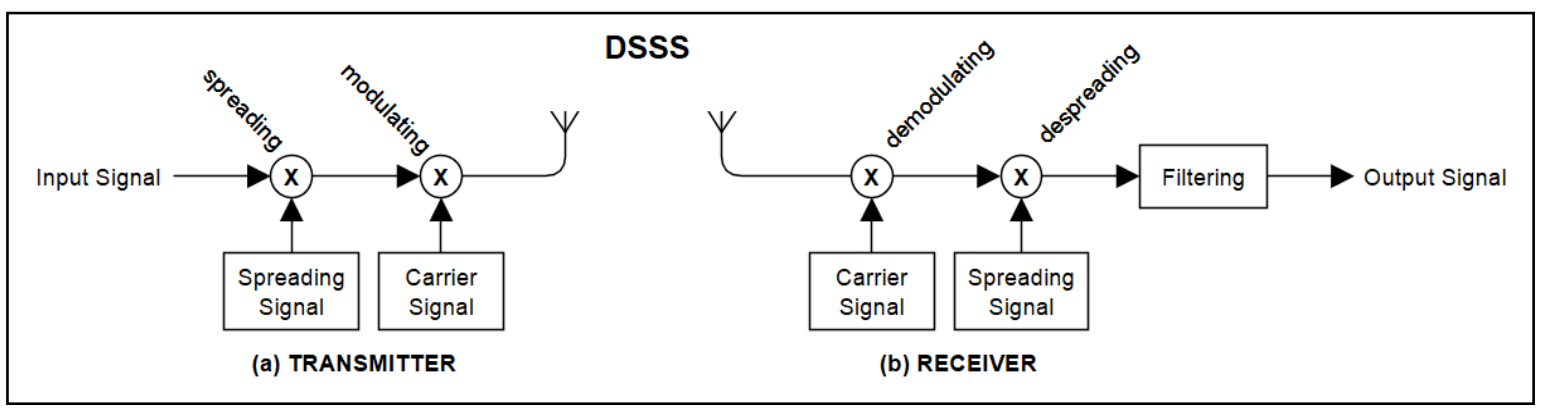

Fig. 2. Block diagram of the (a) transmitter and (b) receiver of the DSSS system

The mathematical model of the transmitted signal from the transmitter of DSSS has the following equation [1]:

$$
T_{D}(t)=d(t) p(t) c(t)
$$

where $T_{D}(t)$ is transmitted signal of DSSS system, $d(t)$ is data signal, $p(t)$ is function of the spreading signal, and $c(t)$ is function of the carrier signal.

The signals $d(t), p(t)$, and $c(t)$ are formulated respectively as follow:

$$
\begin{aligned}
& d(t)=\sum_{i=-\infty}^{\infty} d_{i} \psi\left(t-i T_{d}\right) \\
& p(t)=\sum_{i=-\infty}^{\infty} p_{i} \psi\left(t-i T_{s}\right) \\
& c(t)=\mathrm{A} \cos \left(2 \pi f_{s} t+\theta\right)
\end{aligned}
$$

where $d_{i}$ is data series, $T_{d}$ is data signal period, $p_{i}$ is pseudo noise series, $T_{s}$ is spreading signal period, $A$ is carrier signal amplitude, $\theta$ is carrier signal phase, $f_{s}$ is spreading signal frequency, and $\psi(t-i T)$ is the delayed impulse unit function.

The delayed impulse unit function $\psi(t-i T)$ is simply given by

$$
\psi(t-i T)=\left\{\begin{array}{l}
1,0 \leq(t-i T)<T \\
0, \text { otherwise }
\end{array}\right.
$$

\subsubsection{Frequency Hopping Spread Spectrum}

In FHSS, the frequency of the spreading signal will continue to change or hopping at predetermined time intervals [27]. The transmitter selects the frequency to be used based on the spreading frequency series. Fig. 3 shows the block diagram of transmitter and receiver of FHSS system. From Fig.3, we can notice the main difference between DSSS and FHSS system. In FHSS system, the data signal is firstly modulated with the carrier signal, then spreading it with spreading signal whose frequency is constantly 
changing. The frequency of a spreading signal is selected from a predetermined range of frequencies, and it is known only to the system users. Therefore, the communication process is difficult to disturb if the used frequency of spreading signal is unknown [3].

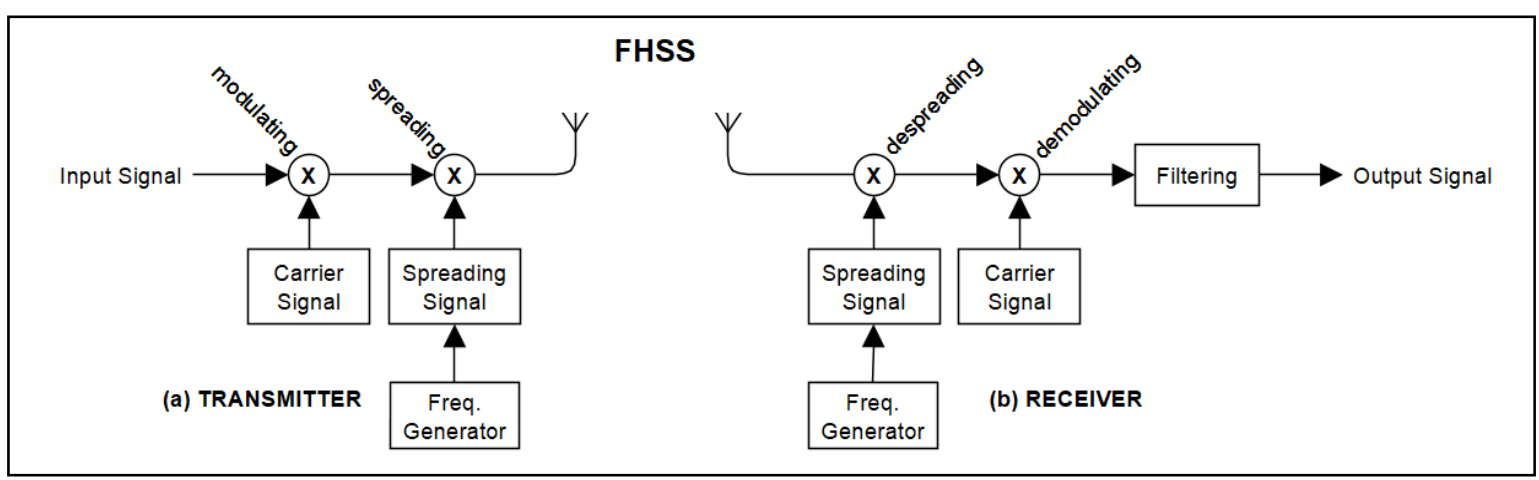

Fig. 3. Block diagram of the (a) transmitter and (b) receiver of the FHSS system

The mathematical equation of the transmitted signal of FHSS is given by [1]:

$$
T_{H}(t)=d(t) c(t) p(t)
$$

where $T_{H}(t)$ is transmitted signal of FHSS system, $d(t)$ is data signal, $p(t)$ is function of the spreading signal, and $c(t)$ is function of the carrier signal.

The data signal $d(t)$ is like DSSS's data signal, while carrier signal $c(t)$ and spreading signal $p(t)$ are different as they are formulated by

$$
\begin{aligned}
& c(t)=A \cos \left(2 \pi f_{d} t+\theta\right) \\
& p(t)=A \cos (2 \pi t \cdot h(t)+\theta)
\end{aligned}
$$

where $f_{d}$ is data signal frequency, $h(t)$ is hopping frequency given by:

$$
h(t)=\sum_{i=-\infty}^{\infty} h_{i} \psi\left(t-i T_{d}\right)
$$

Hopping frequency $h(t)$ is a function of $h_{i}$, in which $h_{i}$ refers to spreading frequency series.

\subsection{Design Procedure for Simulation}

There are two main contributions in this research works i.e.; (1) Simulation and (2) Mathematical analysis. Simulation is performed to simulate the process of sending and receiving signals for both DSSS and FHSS, while Mathematical analysis is done to verify that generated signal at each process is matched with the given mathematical model. The mathematical analysis is also used to justify the performance of both systems when wideband interference is added.

In the simulation, two main signals are generated. The first signal is data signal and the second signal is interference. Simulation procedure for comparing DSSS and FHSS is shown in Fig. 4, and it is called as Comparison program. Comparison program consists of single program that is used to generate Biterror rate (BER) data for each system, in which the program is run 5000 times to get an accurate average of BER data. The output of this program is BER data for every variation of spreading frequency.

BER is defined as the number of high-value digital bits in transmission networks interpreted as low, or otherwise, then divided by the number of bit received or processed over several predefined periods.

$$
\text { BER }=\frac{\# \text { Bit errors }}{\# \text { Total bits }}
$$




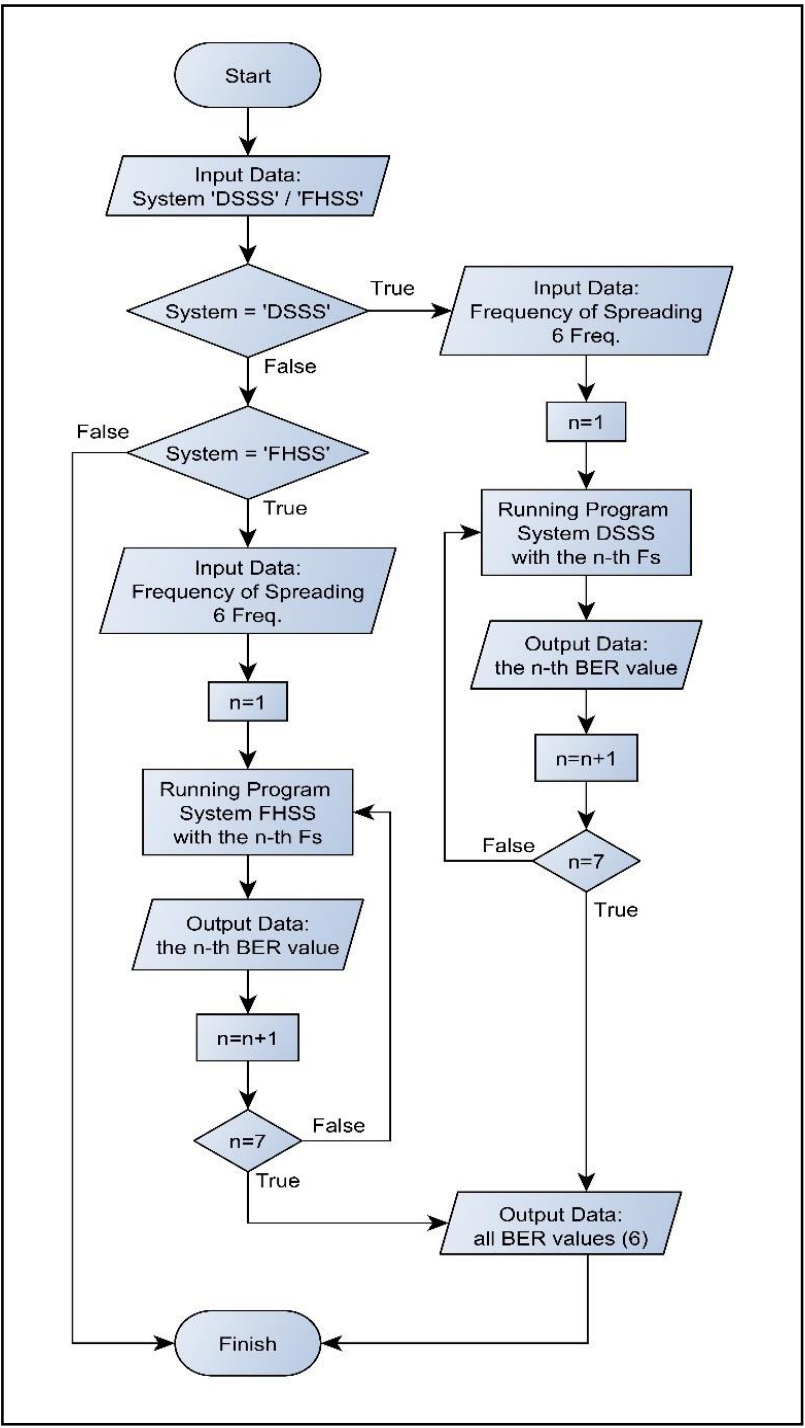

Fig. 4. Comparison Program flow chart

\section{Results and Discussion}

Simulation results comparing the performance of DSSS and FHSS are obtained by running the Comparison Program shown in Fig. 4. By keeping the bitrate at $10 \mathrm{~Hz}$ and varying the spreading frequency, the generated BER for each system will be recorded.

\subsection{DSSS System}

Table 1 shows the simulation results of DSSS system when the bitrate is kept at $10 \mathrm{~Hz}$, while spreading frequency is varied from $40 \mathrm{~Hz}$ to $250 \mathrm{~Hz}$.

Table 1. BER of DSSS system

\begin{tabular}{ccc}
\hline No & Spreading Frequency $(\mathrm{Hz})$ & BER (\%) \\
\hline 1 & 40 & 5.192 \\
2 & 50 & 3.872 \\
3 & 100 & 1.262 \\
4 & 125 & 0.890 \\
5 & 200 & 0.408 \\
6 & 250 & 0.314 \\
\hline
\end{tabular}


Table 1 indicates that there is a change in BER when spreading frequency is varied. When the spreading frequency is increased, the BER value tends to decrease. This behaviour can be explained from the mathematical analysis below. As mentioned before, there are two signals $T_{D}(t)$ and $T_{I}(t)$ generated from Transmitter 1 and 2 respectively. $T_{D}(t)$ is transmitted signal without interference, while $T_{I}(t)$ is interference signal. Fig. 5 demonstrates signal processing steps at the receiver for spreading frequency 40 $\mathrm{Hz}$, while Fig. 6 shows the spectrum of the transmitted signal $T_{D}(t)$ at 3 different spreading frequencies, i.e. $40 \mathrm{~Hz}, 100 \mathrm{~Hz}$, and $200 \mathrm{~Hz}$.

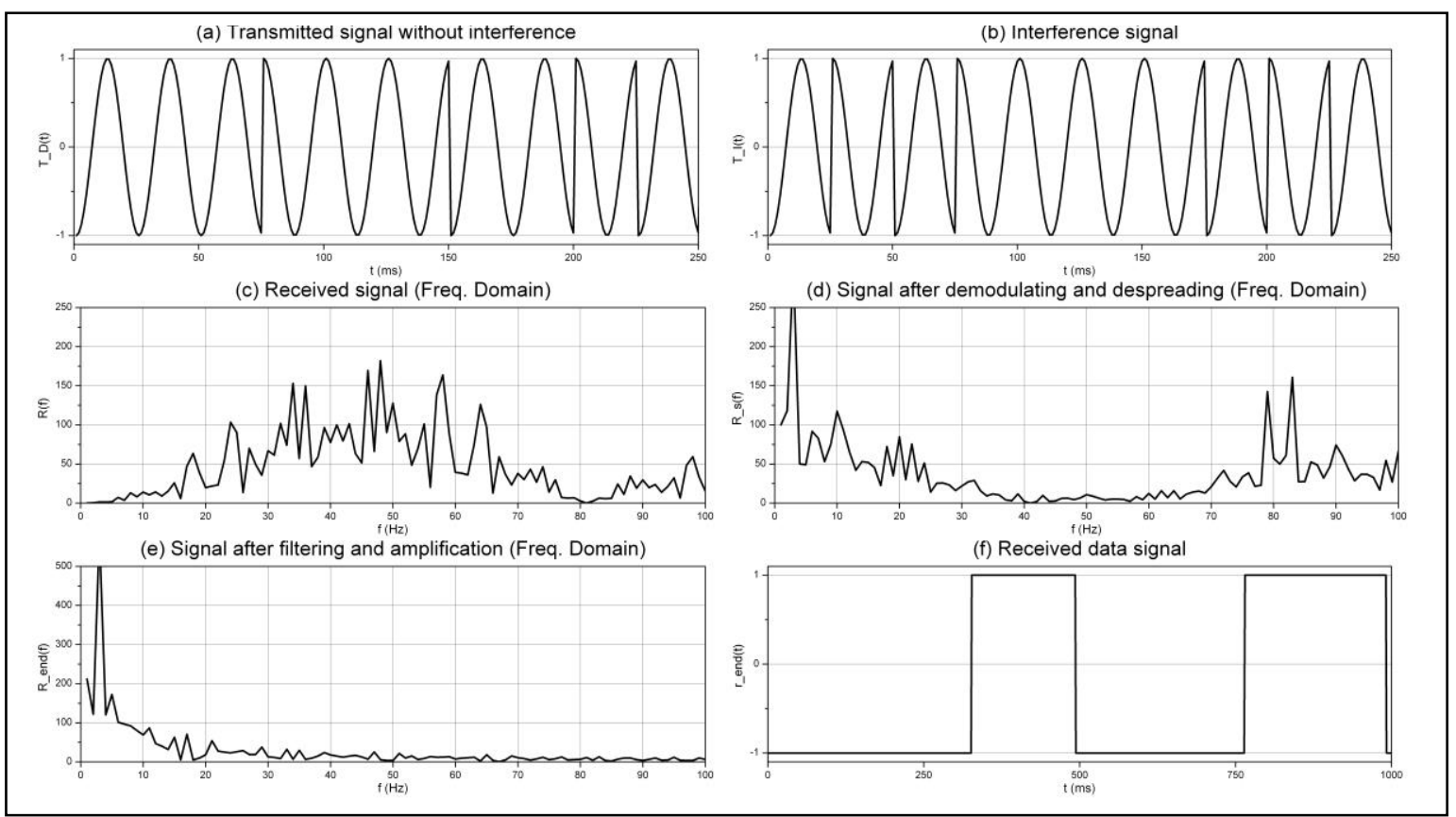

Fig. 5. Signal processing at reciever for DSSS system with spreading frequency $40 \mathrm{~Hz}$

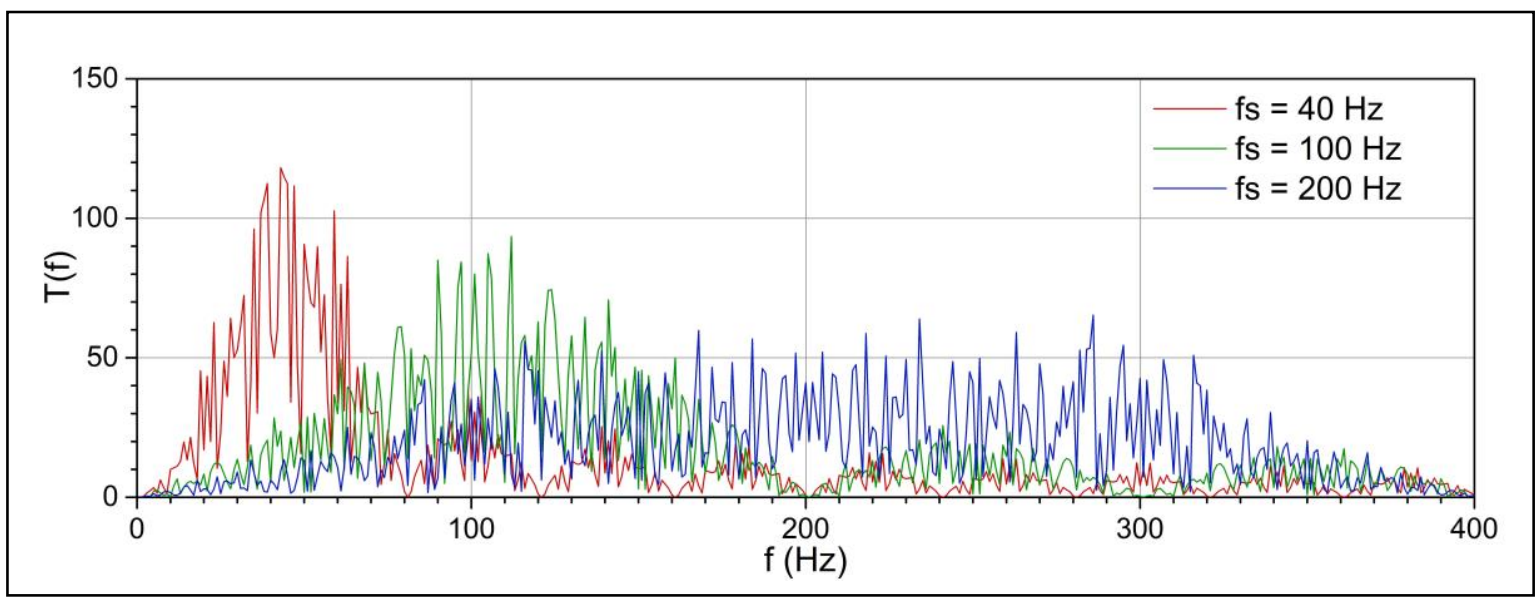

Fig. 6. Spectrum of transmitted signal of DSSS at different spreading frequency

Let $T_{D}(t)$ is as shown in (1), while $T_{I}(t)$ is given by:

$$
T_{I}(t)=d^{\prime}(t) p^{\prime}(t) c(t)
$$

where $c(t)$ is carrier signal shown in (4), $d^{\prime}(t)$ and $p^{\prime}(t)$ are interference data signal and interference spreading signal shown as

$$
\begin{aligned}
& d^{\prime}(t)=\sum_{i=-\infty}^{\infty} d_{i}{ }^{\prime} \psi\left(t-i T_{d}\right) \\
& p^{\prime}(t)=\sum_{i=-\infty}^{\infty} p_{i}{ }^{\prime} \psi\left(t-i T_{s}\right)
\end{aligned}
$$


The interfered data signal of DSSS system is given by:

$$
r(t)=T_{D}(t)+T_{I}(t)=d(t) p(t) c(t)+d^{\prime}(t) p^{\prime}(t) c(t)
$$

The interfered data signal $r(t)$ becomes the received signal at the receiver. Then, the received signal passes through demodulation and de-spreading processes in order to obtain signal $r_{S}(t)$ as follows:

$$
\begin{aligned}
r_{s}(t)= & {\left[\frac{A^{2}}{2} d(t) \cos \left(2 \pi 2 f_{s} t\right)\right]+\left[\frac{A^{2}}{2} d^{\prime}(t) p^{\prime}(t) p(t) \cos \left(2 \pi 2 f_{s} t\right)\right]+\left[\frac{A^{2}}{2} d(t)\right]+} \\
& {\left[\frac{A^{2}}{2} d^{\prime}(t) p^{\prime}(t) p(t)\right] }
\end{aligned}
$$

The signal $r_{s}(t)$ after demodulation and despreading processes will be filtered by using FFT low-pass filter $F(f)$ shown in (16). To recover data signal $d(t)$, the filter bandwidth of $F(f)$ is set to $f_{F}=f_{d}$.

$$
\begin{aligned}
& F(f)=\mu(f)-\mu\left(f-f_{F}\right) \\
& \mu(t)= \begin{cases}1, & t>0 \\
0, & t<0\end{cases}
\end{aligned}
$$

The filtered signal in frequency domain is obtained from:

$$
R_{F}(f)=R_{S}(f) \times F(f)
$$

where $R_{S}(f)$ is $r_{S}(t)$ in frequency domain, and $F(f)$ is filter function.

Thus, signal components having frequency greater than data frequency $f>f_{d}$ or having period smaller than data period $T<T_{d}$, will be eliminated by the filter.

$$
\begin{aligned}
r_{s}(t)= & {\left[\frac{A^{2}}{2}\left(\sum_{i=-\infty}^{\infty} d_{i} \psi\left(t-i \boldsymbol{T}_{\boldsymbol{d}}\right)\right) \cos \left(2 \pi \mathbf{2} \boldsymbol{f}_{\boldsymbol{s}} t\right)\right]+\left[\frac { A ^ { 2 } } { 2 } ( \sum _ { i = - \infty } ^ { \infty } d _ { i } { } ^ { \prime } \psi ( t - i \boldsymbol { T } _ { \boldsymbol { d } } ) ) \left(\sum_{i=-\infty}^{\infty} p_{i}{ }^{\prime} \psi(t-\right.\right.} \\
& \left.\left.\left.i \boldsymbol{T}_{\boldsymbol{s}}\right)\right)\left(\sum_{i=-\infty}^{\infty} p_{i} \psi\left(t-i \boldsymbol{T}_{\boldsymbol{s}}\right)\right) \cos \left(2 \pi \mathbf{2} \boldsymbol{f}_{\boldsymbol{s}} t\right)\right]+ \\
& {\left[\frac{A^{2}}{2}\left(\sum_{i=-\infty}^{\infty} d_{i}{ }^{\prime} \psi\left(t-i \boldsymbol{T}_{\boldsymbol{d}}\right)\right)\left(\sum_{i=-\infty}^{\infty} p_{i}{ }^{\prime} \psi\left(t-i \boldsymbol{T}_{\boldsymbol{s}}\right)\right)\left(\sum_{i=-\infty}^{\infty} p_{i} \psi\left(t-i \boldsymbol{T}_{\boldsymbol{s}}\right)\right)\right]+} \\
& {\left[\frac{A^{2}}{2}\left(\sum_{i=-\infty}^{\infty} d_{i} \psi\left(t-i \boldsymbol{T}_{\boldsymbol{d}}\right)\right)\right] }
\end{aligned}
$$

Given the conditions that $p_{i} \neq p_{i}^{\prime}$ and $f_{s}>f_{d}$, the obtained filtered signal $r_{F}(t)$ is

$$
r_{F}(t) \approx\left[\frac{A^{2}}{2}\left(\sum_{i=-\infty}^{\infty} d_{i} \psi\left(t-i \boldsymbol{T}_{\boldsymbol{d}}\right)\right)\right] \approx \frac{A^{2}}{2} d(t)
$$

The filtered signal $r_{F}(t)$ with $A=1$ will be amplified with gain 2 to obtain signal function that is similar to the data signal $d(t)$

$$
r_{\text {end }}(t) \approx 2 r_{F}(t) \approx d(t)
$$

However, there may be other signal components that can pass through the filtering process, if the following conditions occur:

1. If $f_{s}=f_{d}$ and $p_{i} \neq p_{i}^{\prime}$, then signal components that can pass through the filtering process:

$$
r_{F}(t) \approx\left[\frac{A^{2}}{2} d(t)\right]+\left[\frac{A^{2}}{2} d^{\prime}(t) p^{\prime}(t) p(t)\right]
$$

2. If $f_{s}=0.5 f_{d}$ and $p_{i} \neq p_{i}^{\prime}$, the filtered signal $r_{F}(t)$ is 


$$
\begin{aligned}
r_{F}(t) \approx & {\left[\frac{A^{2}}{2} d(t)\right]+\left[\frac{A^{2}}{2} d(t) \cos \left(2 \pi 2 f_{s} t\right)\right]+\left[\frac{A^{2}}{2} d^{\prime}(t) p^{\prime}(t) p(t)\right]+} \\
& {\left[\frac{A^{2}}{2} d^{\prime}(t) p^{\prime}(t) p(t) \cos \left(2 \pi 2 f_{s} t\right)\right] }
\end{aligned}
$$

3. If $f_{s}>f_{d}$ and $p_{i}^{\prime}=p_{i}$, the filtered signal $r_{F}(t)$ is

$$
r_{F}(t) \approx\left[\frac{A^{2}}{2} d(t)\right]+\left[\frac{A^{2}}{2} d^{\prime}(t)\right]
$$

From the mathematical analysis above, it can be noted that the required conditions for DSSS system to be robust against wideband interference $T_{I}(t)$ are: 1) $f_{s}>f_{d}$, and 2) $p_{i}{ }^{\prime} \neq p_{i}$.

The larger spreading frequency $f_{s}$ compared to bitrate $f_{d}$ will have better robustness due to signal components other than $\frac{A^{2}}{2} d(t)$ will have small chance to pass the filtering process. This mathematical analysis matches with the simulation results shown in Table 1, where the greater the spreading frequency $f_{s}$ to be larger than the bitrate $f_{d}$, then the smaller BER of the system to be obtained.

\subsection{FHSS System}

In the simulation of FHSS system, 6 variations of spreading frequency are simulated. The bitrate is kept at $10 \mathrm{~Hz}$. The largest spreading frequency used in FHSS is equal to the highest spreading frequency used in the DSSS system. Table 2 is the BER results for simulated FHSS system.

Table 2. BER of FHSS system

\begin{tabular}{ccc}
\hline No & Spreading Frequency $(\mathrm{Hz})$ & BER $(\%)$ \\
\hline 1 & 20 and 40 & 3.774 \\
2 & 20 and 50 & 0.126 \\
3 & 20 and 100 & 0.066 \\
4 & 20 and 125 & 0.066 \\
5 & 20 and 200 & 0.064 \\
6 & 20 and 250 & 0.044 \\
\hline
\end{tabular}

There are two hopping frequencies where one of them is kept at $20 \mathrm{~Hz}$, while the other one is increasingly varied to $250 \mathrm{~Hz}$. From Table 2, there is also a change in BER when hopping frequency is increased. When hopping frequency is increased, the BER value becomes smaller. We can also notice that BER of FHSS system is smaller than BER of DSSS system. Fig. 7 shows the signal processing steps at the receiver's side of FHSS. Fig. ${ }_{(\mathrm{f})}$ indicates that data signal is recovered at the end of the process.

Let $T_{H}(t)$ is transmitted signal of FHSS system without interference, while $T_{I}(t)$ is interference signal. $T_{I}(t)$ is given by:

$$
T_{I}(t)=d^{\prime}(t) c(t) p^{\prime}(t)
$$

where $c(t)$ is carrier signal, $d^{\prime}(t)$ is interference data signal, and $p^{\prime}(t)$ is interference spreading signal. The interfered data signal of FHSS system is given by:

$$
r(t)=T_{H}(t)+T_{I}(t)=d(t) c(t) p(t)+d^{\prime}(t) c(t) p^{\prime}(t)
$$

The received signal at the receiver is the interfered data signal $r(t)$. The received signal passes through de-spreading followed by demodulation process. Then, the following signal $r_{m}(t)$ is obtained: 


$$
\begin{aligned}
& r_{m}(t)=\left(A^{2} / 4\right) d(t)+\left(A^{2} / 4\right) d(t) \cos \left(2 \pi 2 f_{d} t\right)+\left(A^{2} / 4\right) d(t) \cos (2 \pi t 2 h(t))+ \\
& \left(A^{2} / 8\right) d(t) \cos \left(2 \pi t 2\left(h(t)-f_{d}\right)\right)+\left(A^{2} / 8\right) d(t) \cos \left(2 \pi t 2\left(h(t)+f_{d}\right)\right)+ \\
& \left(A^{2} / 4\right) d^{\prime}(t) \cos \left(2 \pi t\left(h^{\prime}(t)-h(t)\right)\right)+\left(A^{2} / 4\right) d^{\prime}(t) \cos \left(2 \pi t\left(h^{\prime}(t)+h(t)\right)\right)+ \\
& \left(A^{2} / 8\right) d^{\prime}(t) \cos \left(2 \pi t\left(h^{\prime}(t)-h(t)-2 f_{d}\right)\right)+\left(A^{2} / 8\right) d^{\prime}(t) \cos \left(2 \pi t\left(h^{\prime}(t)-h(t)+2 f_{d}\right)\right)+ \\
& \left(A^{2} / 8\right) d^{\prime}(t) \cos \left(2 \pi t\left(h^{\prime}(t)+h(t)-2 f_{d}\right)\right)+\left(A^{2} / 8\right) d^{\prime}(t) \cos \left(2 \pi t\left(h^{\prime}(t)+h(t)+2 f_{d}\right)\right)
\end{aligned}
$$
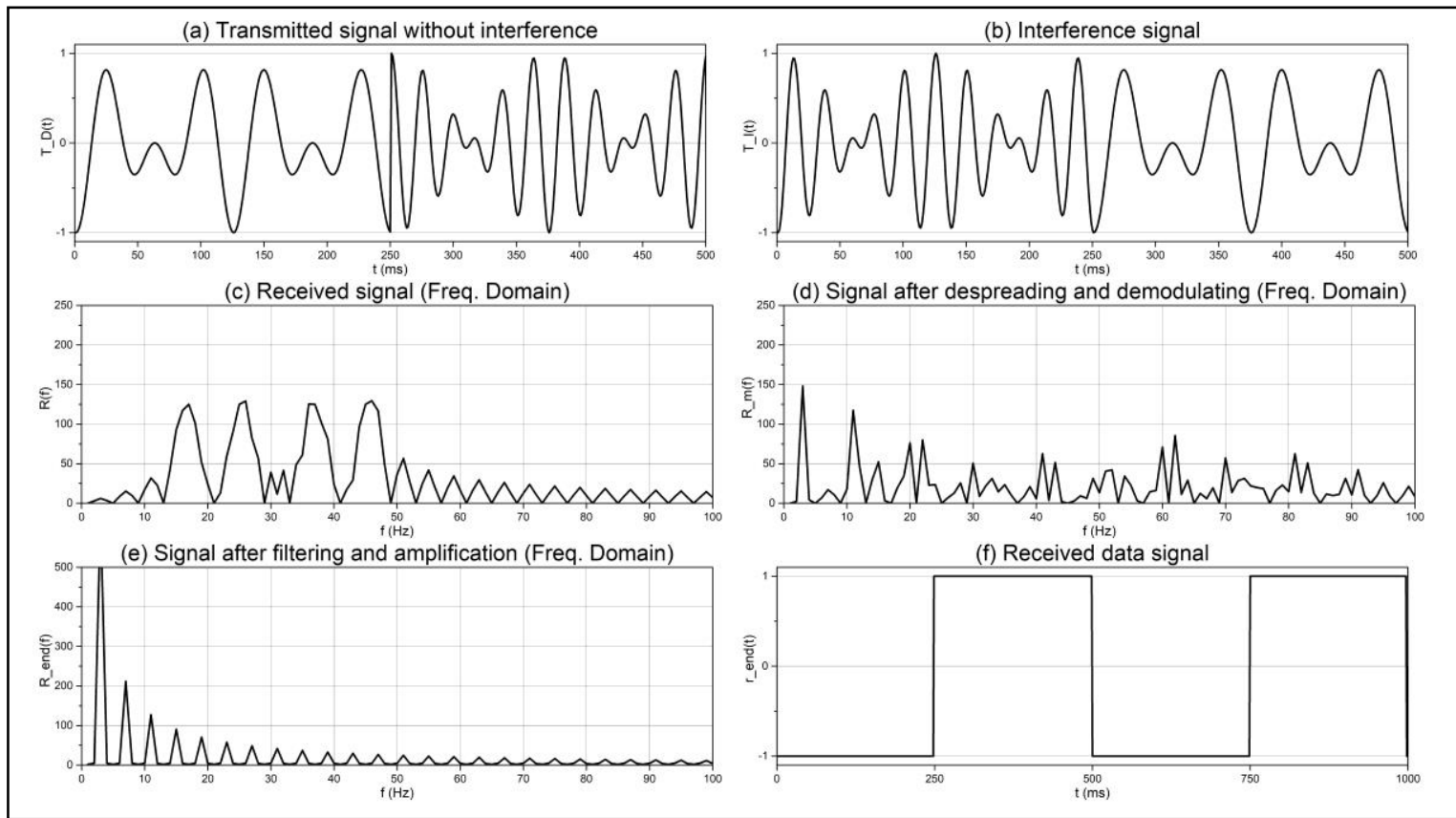

Fig. 7. Signal processing at reciever for FHSS system with hopping frequency 20 and $40 \mathrm{~Hz}$

The signal $r_{m}(t)$ after despreading and demodulation processes will be filtered by using FFT lowpass filter $F(f)$. Filter $F(f)$ is similar to the filter used in DSSS system which has filter bandwidth $f_{d}$. Given the conditions that; (a) $h_{i} \neq h_{i}{ }^{\prime}$, (b) $h_{i}>\frac{3}{2} f_{d}$, (c) $h_{i}{ }^{\prime}>\frac{3}{2} f_{d}$ and (d) $\left|h_{i}-h_{i}^{\prime}\right|>3 f_{d}$, then the filtered signal $r_{F}(t)$ below is obtained:

$$
r_{F}(t) \approx\left[\frac{A^{2}}{4}\left(\sum_{i=-\infty}^{\infty} d_{i} \psi\left(t-i \boldsymbol{T}_{\boldsymbol{d}}\right)\right)\right] \approx \frac{A^{2}}{4} d(t)
$$

For FHSS system, the filtered signal $r_{F}(t)$ with $A=1$ will be amplified with gain 4 to recover the data signal $d(t)$.

$$
r_{\text {end }}(t) \approx 4 r_{F}(t) \approx d(t)
$$

Like DSSS system, there may be other signal components that can pass through the filtering process, if the following conditions happen:

1. If $h_{i}=\frac{3}{2} f_{d} ;\left|h_{i}-h_{i}^{\prime}\right|>3 f_{d} ; h_{i}{ }^{\prime}>\frac{9}{2} f_{d} ; h_{i}^{\prime} \neq h_{i}$, then the filtered signal $r_{F}(t)$ is

$$
r_{F}(t) \approx\left[\frac{A^{2}}{4} d(t)\right]+\left[\frac{A^{2}}{8} d(t) \cos \left(2 \pi t f_{d}\right)\right]
$$

2. If $h_{i}=\frac{1}{2} f_{d},\left|h_{i}-h_{i}^{\prime}\right|>3 f_{d} ; h_{i}{ }^{\prime}>\frac{7}{2} f_{d} ; h_{i}^{\prime} \neq h_{i}$, then the filtered signal $r_{F}(t)$ is 


$$
r_{F}(t) \approx\left[\frac{A^{2}}{4} d(t)\right]+\left[\frac{3 A^{2}}{8} d(t) \cos \left(2 \pi t f_{d}\right)\right]
$$

3. If $h_{i}^{\prime}=h_{i} ;\left|h_{i}-h_{i}{ }^{\prime}\right|=0 ; h_{i}>\frac{3}{2} f_{d} ; h_{i}{ }^{\prime}>\frac{3}{2} f_{d}$, then the filtered signal $r_{F}(t)$ is

$$
r_{F}(t) \approx\left[\frac{A^{2}}{4} d(t)\right]+\left[\frac{A^{2}}{4} d^{\prime}(t)\right]
$$

From the mathematical analysis above, it can be concluded that FHSS system is robust against this type of interference if the following conditions are met: 1) $h_{i}{ }^{\prime} \neq h_{i}$;2) $h_{i}>\frac{3}{2} f_{d}$;3) $h_{i}{ }^{\prime}>\frac{3}{2} f_{d}$; and 4) $\left|h_{i}-h_{i}^{\prime}\right|>3 f_{d}$.

As explained before, $h_{i}$ is spreading frequency series of the data signal. Here, $h_{i}{ }^{\prime}$ is spreading frequency series of interference. The required conditions $h_{i}>\frac{3}{2} f_{d}$ and $h_{i}^{\prime}>\frac{3}{2} f_{d}$ match to the trend of the obtained BER data shown in Table 2. If the hopping frequency (spreading frequency series) is greater than 1.5 times of the bitrate, then we get small BER. And, if we keep increasing the spreading frequency series, then BER value gets smaller indicating the system performance becomes more optimal.

When the hopping frequency are varied from a pair of 20 and $40 \mathrm{~Hz}$ to 20 and $100 \mathrm{~Hz}$, we can see a significant change in BER value. This can be understood from the fact that condition of $\left|h_{i}-h_{i}^{\prime}\right|>$ $3 f_{d}$ has been satisfied. Let assess the first case when the hopping frequency are 20 and $40 \mathrm{~Hz}$. The spreading frequency series of the data signal will be $h_{1}=20 \mathrm{~Hz}$ and $h_{2}=40 \mathrm{~Hz}$, while the spreading frequency series of the interference will be in vice versa, $h_{1}{ }^{\prime}=40 \mathrm{~Hz}$ and $h_{2}{ }^{\prime}=20 \mathrm{~Hz}$. Thus, the absolute difference between $h_{i}$ and $h_{i}^{\prime}$ will be less than $3 f_{d}$, or, $\left|h_{i}-h_{i}^{\prime}\right|<3 f_{d}$. Hence, this does not meet condition 4, while conditions 1 to 3 are still met. At these frequency hopping, the resulted BER value is $3.774 \%$.

Let also assess the second case when the hopping frequency $20 \mathrm{~Hz}$ and $100 \mathrm{~Hz}$. The $h_{1}$ and $h_{2}$ will be 20 and $100 \mathrm{~Hz}$, while $h_{1}{ }^{\prime}$ and $h_{2}{ }^{\prime}$ are 100 and $20 \mathrm{~Hz}$ respectively. Thus, the absolute difference between $h_{i}$ and $h_{i}^{\prime}$ is larger than $3 f_{d}$, or, $\left|h_{i}-h_{i}^{\prime}\right|>3 f_{d}$. Hence, all the required conditions (1 to 4$)$ are satisfied which results in very small BER. In this case, the resulted BER value is $0.066 \%$ which is far smaller compared to the first case.

\subsection{Remarks}

After simulation tests and mathematical analysis have been performed, we can notice the ability of each system under wideband interference. We can point out that FHSS has a better performance compared to DSSS. Here, the following important remarks can be proposed;

(1) DSSS system has two conditions; 1) $f_{s}>f_{d}$, and 2) $p_{i}{ }^{\prime} \neq p_{i}$. The first condition, $f_{s}>f_{d}$, is general requirement for DSSS system. The second condition, ${p_{i}}^{\prime} \neq p_{i}$, means that the pseudo noise series of the data signal must be different to the pseudo noise series of the interference signal. Where it only happens if:

$$
\begin{array}{ll}
\text { Suppose: } & p_{i}=1,1,0,0,1,0,1,0 \ldots \\
\text { Then: } & p_{i}^{\prime}=0,0,1,1,0,1,0,1 \ldots
\end{array}
$$

Both $p_{i}$ and $p_{i}{ }^{\prime}$ are binary data which are randomly generated by the system. Therefore, there may be one and more similar pseudo noise series between $p_{i}$ and $p_{i}{ }^{\prime}$. Hence, the chance for $p_{i}{ }^{\prime} \neq p_{i}$ is $50 \%$. This is one of the reasons that makes DSSS is less optimal than FHSS.

(2) FHSS has four conditions; 1) $\left.\left.h_{i}>\frac{3}{2} f_{d}, 2\right) h_{i}{ }^{\prime}>\frac{3}{2} f_{d}, 3\right) h_{i}{ }^{\prime} \neq h_{i}$, and 4) $\mid h_{i}-h_{i}{ }^{\prime}>3 f_{d}$. The first and second conditions, $h_{i}>\frac{3}{2} f_{d}$ and $h_{i}{ }^{\prime}>\frac{3}{2} f_{d}$, are compulsory requirement in FHSS. These two conditions are like $f_{s}>f_{d}$ in DSSS system. The condition $h_{i}{ }^{\prime} \neq h_{i}$ is similar to $p_{i}{ }^{\prime} \neq p_{i}$ in DSSS system. However, the possibility for $h_{i}{ }^{\prime} \neq h_{i}$ in FHSS is greater than $p_{i}{ }^{\prime} \neq p_{i}$ in DSSS system. This is due to the spreading frequency series $h_{i}$ is a type of positive decimal data $\left(h_{i}>0\right)$, while $p_{i}$ is a type of binary data $\left(p_{i}=0\right.$ or 1$)$. Moreover, the condition $\left|h_{i}-h_{i}^{\prime}\right|>3 f_{d}$ has a greater possibility, because if $h_{i} \gg f_{d}$ then the absolute difference between $h_{i}$ and $h_{i}^{\prime}$ will also be much greater than $f_{d}$. 


\section{Conclusion}

In this paper, performance evaluation and mathematical analysis of DSSS and FHSS systems subject to wideband interference have been presented. Simulation has been performed to evaluate the performance of both systems when the spreading frequencies are varied. Simulation results demonstrate that BER of both systems are decreased, when spreading frequencies are increased. Mathematical analysis verifies the performance of each system, where the optimum conditions for each system has also been demonstrated. It has been proposed that DSSS requires two conditions, while FHSS requires 4 conditions, to meet its optimum condition against wideband interference. Based on simulation results and mathematical analysis, FHSS is more optimal than DSSS, especially when the system is subject to wideband interference.

\section{References}

[1] D. Torrieri, Principles of Spread-Spectrum Communication Systems, Maryland: Springer International Publishing Switzerland, 2015, available at: https://ink.springer.com/book/10.1007\%2F978-3-319-70569-9.

[2] R. 1. Pickholtz, D. 1. Schilling and L. B. Milstein, "Theory of Spread-Spectrum Communications," IEEE Transactions on Communications, vol. 30, no. 5, pp. 855-884, 1982, doi : https://doi.org/10.1109/ TCOM.1982.1095533.

[3] T. Tsui and T. Clarkson, "Spread-Spectrum Communication Techniques," Electronics \& Communication Engineering Journal, vol. 6, no. 1, pp. 3-12, 1994, doi : https://doi.org/10.1049/ecej:19940101.

[4] G. Ponuratinam, B. Patel, S. S. Rizvi and K. M. Elleithy, "Improvement in the Spread Spectrum System in DSSS, FHSS, AND CDMA," in ASEE, Bridgeport, 2009, available at : https://scholarworks.bridgeport.edu/ xmlui/handle/123456789/741.

[5] K. Wesołowski, Introduction To Digital Communication Systems, West Sussex: John Wiley \& Sons Ltd, 2009, available at : Google Scholar.

[6] E. Cianca and R. Prasad, "Spread Spectrum Techniques and their Applications to Wireless Communications," IETE Journal of Research, vol. 51, no. 1, pp. 5-18, 2005, doi : https://doi.org/10.1080/ 03772063.2005.11416373.

[7] A. Jamalipour and A. Ogawa, "Packet admission control in a direct-sequence spread-spectrum LEO satellite communications network," IEEE Journal on Selected Areas in Communications, vol. 15, no. 8, pp. 1649-1656, 1997, doi : https://doi.org/10.1109/49.634802.

[8] J. Meel, "Spread Spectrum (SS) applications," De Nayer Instituut, Hogeschool Voor Wetenschap \& Kunst, Rotselaar, 1999, available at : https://www.csie.ntu.edu.tw/ b6506031/ExpReport/appl_ss.pdf..pdf.

[9] S. V. Maric, Z. I. Kostic and E. L. Titlebaum, "A new family of optical code sequences for use in spreadspectrum fiber-optic local area networks," IEEE Transactions on Communications, vol. 41, no. 8, pp. 12171221, 1993, doi : https://doi.org/10.1109/26.231965.

[10] M. Ednch and R. Schmalenberger, "Combined DSSS/FHSS approach to interference rejection and navigation support in UAV communications and control," in IEEE Seventh International Symposium on Spread Spectrum Techniques and Applications, Prague, 2002, doi : https://doi.org/10.1109/ISSSTA. 2002.1049347.

[11] F. Qu, X. Qin, L. Yang and T. C. Yang, "Spread-Spectrum Method Using Multiple Sequences for Underwater Acoustic Communications," IEEE Journal of Oceanic Engineering, vol. PP, no. 99, pp. 1-12, 2017, doi : https://doi.org/10.1109/JOE.2017.2750298.

[12] I. V. Bolo, T. P. Espera, R. V. Marquez, C. D. Ambatali and N. I. Bernardo, "Performance Evaluation of Spread Spectrum-based Multiple Access Combined with 5G Filter-based Multi-carrier Waveforms," Institute of Electrical and Electronics Engineers, 2017, doi : https://doi.org/10.1109/ICSPCS.2017.8270475.

[13] Y. Xiang, I. Natgunanathan, D. Peng, G. Hua and B. Liu, "Spread Spectrum Audio Watermarking Using Multiple Orthogonal PN Sequences and Variable Embedding Strengths and Polarities," IEEE/ACM Transactions on Audio, Speech, and Language Processing, vol. 26, no. 3, pp. 529-539, 2018, doi : https://doi.org/ 10.1109/TASLP.2017.2782487.

[14] I. J. Cox, J. Kilian, T. Leighton and T. Shamoon, "Secure spread spectrum watermarking for images, audio and video," Institute of Electrical and Electronics Engineers, pp. 243-246, 1996, doi : https://doi.org/10.1109/ ICIP.1996.560429. 
[15] I. J. Cox, J. Kilian, F. T. Leighton and T. Shamoon, "Secure Spread Spectrum Watermarking for Multimedia," IEEE Transactions on Image Processing, vol. 6, no. 12, pp. 1673-1687, 1997, doi : https://doi.org/10.1109/83.650120.

[16] A. Singh, B. Kumar, M. Dave and . Mohan, "Robust and Imperceptible Spread-Spectrum Watermarking for Telemedicine Applications," Proceedings of the National Academy of Sciences, India Section A: Physical Sciences, vol. 85, no. 2, pp. 295-301, 2015, doi : https://doi.org/10.1007/s40010-014-0197-6.

[17] F. Pareschi, R. Rovatti and G. Setti, "EMI Reduction via Spread Spectrum in DC/DC Converters: State of the Art, Optimization, and Tradeoffs," IEEE Access, vol. 3, pp. 2857-2874, 2015, doi : https://doi.org/ 10.1109/ACCESS.2015.2512383.

[18] H.-P. Park, M. Kim and J.-H. Jung, "Spread Spectrum Technique to Reduce EMI Emission for an LLC Resonant Converter Using a Hybrid Modulation Method," IEEE Transactions on Power Electronics, vol. 33, no. 5, pp. 3717-3721, 2018, doi : https://doi.org/10.1109/TPEL.2017.2766284.

[19] H. Alsuraisry, J. Cheng, J. Lin, Y. Kuo, J. Tsai and T. Huang, "A 6-GHz spread spectrum clock generation with EMI reduction of $30.2 \mathrm{~dB}$ for SATA-III applications," Microwave and Optical Technology Letters, vol. 59, no. 3, pp. 622-624, 2017, doi : https://doi.org/10.1002/mop.30359.

[20] R. 1. Pickholtz, L. B. Milstein and D. 1. Schilling, "Spread Spectrum for Mobile Communications," IEEE Transactions On Vebicular Technology, vol. 40, no. 2, pp. 313-322, 1991, doi : https://doi.org/10.1109/ 25.289412 .

[21] W. Xiaoying, "Study Spread Spectrum In Matlab," School of Electrical \& Electronic Engineering, Nanyang Technological University, Nanyang Drive, 2003, available at : https://pdfs.semanticscholar.org/1305/ 72bc14138636b6beae5ce6a74df8a36a44da.pdf.

[22] P. Jung, P. W. Baier and A. Steil, "Advantages of CDMA and spread spectrum techniques over FDMA and TDMA in cellular mobile radio applications," IEEE Transactions on Vebicular Technology, vol. 42, no. 3, pp. 357-364, 1993, doi : https://doi.org/10.1109/25.231889.

[23] S. Thuneibat, "Direct Sequence Spread Spectrum with Barker Code and QPSK," IOSR Journal of Engineering , vol. 06, no. 06, pp. 62-67, 2016, available at : https://pdfs.semanticscholar.org/7a63/cb112646f8a61c5bff55 66e6e8c1fcecb533.pdf.

[24] P. Olšovský and P. Podhoranský, "Design And Simulation Of Frequency Hopping Technique In Matlab," Institute of Electronics and Photonics, Faculty of Electrical Engineering and Information Technology, Slovak University of Technology, Bratislava, available at : http://www.humusoft.cz/ftp/www/papers/tcb12/059_ olsovsky.pdf.

[25] S. S. S. Sugi and V. J. C, "Spread Spectrum Modulation Techniques using MATLAB," International Journal Of Innovative Research In Technology, vol. 2, no. 3, pp. 47-52, 2015, available at : http://ijirt.org/master/ publishedpaper/IJIRT142537_PAPER.pdf.

[26] P. Waghmare and R. Nawkhare, "Performance Evaluation of DSSS and FHSS Using Modulation Technique," International Journal of Innovative Research in Computer and Communication Engineering, vol. 3, no. 10, pp. 9150-9156, 2015, available at : https://pdfs.semanticscholar.org/9464/689125eec7e8c3f017563 84011a01f9a7552.pdf.

[27] L. Nurmalia and M. Pinem, "Analisis Perbandingan Teknologi Spread Spectrum FHSS dan DSSS pada Sistem CDMA," SINGUDA ENSIKOM, vol. 4, no. 1, pp. 30-35, 2013, available at : https://jurnal.usu.ac.id/ index.php/singuda_ensikom/article/view/3678/2299.

[28] M. Soroushnejad and E. Geraniotis, "Probability of capture and rejection of primary multiple-access interference in spread-spectrum networks," IEEE Transactions on Communications, vol. 39, no. 6, pp. 986994, 1991, doi : https://doi.org/10.1109/26.87188.

[29] E. McCune, "DSSS vs. FHSS narrowband interference performance issue," RF Signal Processing, 2000, available at : https://pdfs.semanticscholar.org/a7e0/95d9613e511c757021c41cf579af404bc9bb.pdf.

[30] M. G. Shayesteh and M. N. Kenari, "Multiple-Access Performance Analysis of Combined Time-Hopping and Spread-Time CDMA System in the Presence of Narrowband Interference," IEEE Transactions on Vebicular Technology, vol. 58, no. 3, pp. 1315-1328, 2009, doi : https://doi.org/10.1109/TVT.2008.927723. 